\title{
Editorial to the special issue Synergies Between Solar and Stellar Modelling
}

\author{
M.P. Di Mauro • M. Marconi • D. Cardini
}

Received: 27 April 2010 / Accepted: 27 April 2010 / Published online: 10 June 2010

(C) Springer Science+Business Media B.V. 2010

The theory of stellar evolution allows us to interpret the observed properties of the Sun and of the other stars in the Milky Way, as well as in other galaxies resolved in stars. However, several open problems in our understanding of stellar structures, and of their role in the galactic formation and evolution mechanisms, are due to limitations of the theoretical evolutionary framework, that does not account for internal dynamics.

The possibility to explore the inner structure of stars through the investigation of the seismic waves observed at their surfaces, has become one of the most challenging opportunity of modern astrophysics and a crucial benchmark for theoreticians. Since the discovery of solar oscillations, extensive ground-based networks have been devoted to the observation of this phenomenon, and thanks also to the results from the SOHO satellite and to the development of powerful theoretical seismological tools, Helioseismology has revealed the internal structure of the Sun, thus transforming our star into a true cosmic physics laboratory. The extension of this approach to other stars through the advent of the astroseismological space missions (e.g. MOST, COROT, KEPLER), the organization of modern devoted ground-based projects and the application of theoretical tools typically developed for the Sun to stars with dif-

M.P. Di Mauro $(\varangle) \cdot$ D. Cardini

INAF-IASF Roma, Istituto di Astrofisica Spaziale e Fisica

Cosmica, via del Fosso del Cavaliere 100, 00133 Rome, Italy

e-mail: mariapia.dimauro@iasf-roma.inaf.it

D. Cardini

e-mail: daniela.cardini@iasf-roma.inaf.it

M. Marconi

INAF-Osservatorio Astronomico di Capodimonte,

Via Moiariello 16, 80131 Napoli, Italy

e-mail:marcella@na.astro.it ferent mass, chemical composition and evolutionary phase is the crucial subsequent important step.

This volume presents the state of the art in the solar and stellar modelling researches focusing on the most relevant synergies between the two approaches, as well as on the perspectives for the next decades, to disclose several important still open questions.

The structure of the stars cannot be so well constrained such as that of the Sun. The main difference between solar and stellar modelling obviously depends on the fact that the global parameters of the Sun are much better known than they are for any other star. Luminosity, effective temperature, surface composition and rotational velocity are obtained, within a certain error, from spectrum analysis of distant stars; age and composition are approximately estimated only for stars in clusters; masses and radii are measured only for spectroscopic binaries. In addition, all these measurements can be affected by unknown effects such as loss, accretion, or diffusion of mass.

Particular emphasis is devoted to the role of the input physics, and its relevant uncertainties, in the construction of stellar models and in the resulting predictions for general observable quantities. The result about the new determinations of the solar photospheric abundances and its implication for solar and stellar models are well discussed. Issues related to convection, overshoot, diffusion and settling of helium and heavy elements, rotation, chemical composition and magnetic field are extensively discussed and the effects of these phenomena properly explored and quantified in view of an exhaustive two or three-dimensional representation of stars.

Much space in this volume is dedicated to the application of helio- and asteroseismic techniques as tools to test the predictions of the theory of stellar structure and evolution as well as stellar atmosphere models. Comments on prospects for future improvements and refinements of the 
theoretical models are given, focusing on the possibility of getting ever more precise helioseismic and asteroseismic observations from ground and space.

The articles published in this issue are arranged in eight topical groups:

- Solar and stellar models

- Helio- and asteroseismology: diagnostic and techniques

- Equation of state and nuclear reactions

- The chemical composition

- Convection, overshooting and the excitation of modes

- Solar and stellar rotation
- Solar and stellar magnetic field

- Future observational projects in helio- and asteroseismology

Finally, we would like to acknowledge the European Helioand Asteroseismology Network (HELAS - www.helas-eu. org), a major European collaboration funded by the European Commission under FP6 (contract FP6-2004-Infrastructures-5-026138), which strongly supported the publication of this volume. 